\title{
Dynamics of a Stage-Structured Predator-Prey Model
}

\author{
Sunita Gakkhar*, Komal Gupta \\ Department of Mathematics, Indian Institute of Technology Roorkee, Uttarakhand, India. \\ * Corresponding author. Tel.: +91-9719242545; email: sungkfma@gmail.com \\ Manuscript submitted April 24, 2016; accepted September 29, 2016. \\ doi: 10.17706/ijapm.2017.7.1.24-32
}

\begin{abstract}
This paper deals with the dynamics of a stage-structured predator-prey system. The immature and mature prey are predated by the predator for which modified Holling type II functional response is considered in the model. The solution of the system is positive and bounded. Stability analysis has been discussed about all possible feasible equilibrium points. The origin and boundary equilibrium points are shown to be globally asymptotically stable. The parameters are identified for which system also admits trans-critical bifurcation about these points. The occurrence of Hopf bifurcation has been shown through numerical simulation about positive interior point. Persistence condition is obtained.
\end{abstract}

Key words: Global stability, Hopf bifurcation, persistence, trans-critical bifurcation.

\section{Introduction}

In the real world, almost all animals have the stage structure of immature and mature population. It has its own significance in the dynamics of the interacting species. Many authors have studied the stage-structured predator-prey models, in which either juvenile prey or adult prey is predated by the predator [1]-[5]. Some authors have investigated stage-structured predator-prey systems where predator is predating over juvenile/ immature or adult/ mature prey [6], [7]. A predator-prey system with group defense of immature class is considered, in which it is proved that for stable co-existence point, mortality rate of mature prey and carrying capacity must be neither too small nor too large [6].

Yang and Zhong [7] have considered two stage-structured deterministic and stochastic predator-prey systems where juvenile prey is predated with Beddington-DeAngel is functional response, while adult prey is predator with Holling-type II functional response. In this model, it has been assumed that the energy derived from immature prey is independent of mature prey. It does not matter that how much the predator has already taken from immature while predating over mature prey. This is possible if predator has two independent guts; one for immature prey and other for mature prey. However, this is not true. To resolve this issue, modified Holling type functional response is proposed, where the functional response incorporates both immature and mature population.

\section{Model Formulation}

Let $X_{1}(t), X_{2}(t)$ and $Y(t)$ be the densities of immature prey, mature prey and predator respectively. Growth of juvenile prey species is proportional to mature prey. The predator is predating over both types of prey with modified Holling type II functional response. The predator-prey system with stage structure can be modeled as follows: 


$$
\begin{aligned}
& \frac{d X_{1}}{d T}=R X_{2}-D_{1} X_{1}-C X_{1}-\frac{A_{1} X_{1} Y}{B_{0}+B_{1} X_{1}+B_{2} X_{2}} \\
& \frac{d X_{2}}{d T}=C X_{1}-D_{2} X_{2}-D_{2}^{\prime} X_{2}^{2}-\frac{A_{2} X_{2} Y}{B_{0}+B_{1} X_{1}+B_{2} X_{2}} ; \quad R_{+}^{3}=\left\{X_{1} \geq 0, X_{2} \geq 0, Y \geq 0\right\} \\
& \frac{d Y}{d T}=-D_{3} Y+\frac{\left(A_{1}^{\prime} X_{1} Y+A_{2}^{\prime} X_{2} Y\right)}{B_{0}+B_{1} X_{1}+B_{2} X_{2}} \\
& \text { with } X_{1}(0)=X_{10} \geq 0, X_{2}(0)=X_{20} \geq 0, Y(0)=Y_{0} \geq 0
\end{aligned}
$$

The growth of the immature species directly depends upon mature prey and $D_{1}, D_{2}$ and $D_{3}$ are the death rates of immature, mature prey and predator respectively. Let $R$ be the birth rate and $C$ be the conversion rate of immature prey. The crowing effect is considered only on the adult prey and is denoted by $D_{2}^{\prime}$.

Let us introduce non-dimensional state variables and parameters as below:

$$
\begin{aligned}
& t=\left(D_{1}+C\right) T, x_{1}=\frac{A_{2}^{\prime}}{R} X_{1}, x_{2}=\frac{A_{2}^{\prime}}{\left(D_{1}+C\right)} X_{2}, y=\frac{A_{2}}{\left(D_{1}+C\right)} Y, \alpha_{1}=\frac{A_{1}}{A_{2} B_{0}}, \alpha_{2}=\frac{1}{B_{0}}, \alpha_{1}^{\prime}=\frac{A_{1}^{\prime} R}{A_{2}^{\prime} B_{0}\left(D_{1}+C\right)} \\
& \beta_{1}=\frac{C R}{\left(D_{1}+C\right)^{2}}, \beta_{2}=\frac{D_{2}}{\left(D_{1}+C\right)}, \beta_{3}=\frac{D_{2}^{\prime}}{A_{2}^{\prime}}, \beta_{4}=\frac{D_{3}}{\left(D_{1}+C\right)}, \delta_{1}=\frac{B_{1} R}{A_{2}^{\prime} B_{0}}, \delta_{2}=\frac{B_{2}\left(D_{1}+C\right)}{A_{2}^{\prime} B_{0}}
\end{aligned}
$$

Then the dimensionless form of the system (1) is given as

$$
\begin{aligned}
& \frac{d x_{1}}{d t}=x_{2}-x_{1}-\frac{\alpha_{1} x_{1} y}{1+\delta_{1} x_{1}+\delta_{2} x_{2}} \\
& \frac{d x_{2}}{d t}=\beta_{1} x_{1}-\beta_{2} x_{2}-\beta_{3} x_{2}^{2}-\frac{\alpha_{2} x_{2} y}{1+\delta_{1} x_{1}+\delta_{2} x_{2}} ; R_{+}^{3}=\left\{x_{1} \geq 0, x_{2} \geq 0, y \geq 0\right\} \\
& \frac{d y}{d t}=-\beta_{4} y+\frac{\left(\alpha_{1}^{\prime} x_{1} y+\alpha_{2} x_{2} y\right)}{1+\delta_{1} x_{1}+\delta_{2} x_{2}} \\
& \text { with } x_{1}(0)=x_{10} \geq 0, x_{2}(0)=x_{20} \geq 0, y(0)=y_{0} \geq 0
\end{aligned}
$$

\section{Analysis}

From the biological point of view, it is necessary to establish that the solution of the initial value problem (2) remains positive and bounded.

It may be observed that $x_{1}-x_{2}$ plane is invariant since $\dot{y}=0$. Further, $\dot{x}_{1}>0, \dot{x}_{2}<0$ on $x_{2}-y$ plane while $\dot{x}_{1}<0, \dot{x}_{2}>0$ on $x_{1}-y$ plane. Accordingly, the vector fields point towards the interior of $R_{+}^{3}$.

To establish the boundedness of the system, consider the function

$$
\Psi(t)=\frac{\alpha_{1}^{\prime}}{\alpha_{1}} x_{1}(t)+x_{2}(t)+y(t)
$$

Computing its time derivative and substitution of (2) and further simplification yields 


$$
0<\Psi \leq \frac{\alpha_{1}^{\prime 2}}{4 \alpha_{1}^{2} \beta_{3} \eta} \text { where } \eta=\min \left\{\left(1-\frac{\beta_{1} \alpha_{1}}{\alpha_{1}^{\prime}}\right), \beta_{2}, \beta_{4}\right\}
$$

\subsection{Existence of Equilibrium Points}

The stage structured predator-prey system (2) admits the following three equilibrium points:

1) The trivial equilibrium point $E_{0}=(0,0,0)$ always exists.

2) The planar equilibrium point $E_{1}=\left(\frac{\beta_{1}-\beta_{2}}{\beta_{3}}, \frac{\beta_{1}-\beta_{2}}{\beta_{3}}, 0\right)$ exists, provided $\beta_{1}>\beta_{2}$

3) The non trivial point $E^{*}=\left(x_{1}^{*}, x_{2}^{*}, y^{*}\right)$, where $x_{1}^{*}, x_{2}^{*}$ and $y^{*}$ can be obtained by solving following three

equations:

$$
\begin{aligned}
& x_{2}-x_{1}-\frac{\alpha_{1} x_{1} y}{1+\delta_{1} x_{1}+\delta_{2} x_{2}}=0 \\
& \beta_{1} x_{1}-\beta_{2} x_{2}-\beta_{3} x_{2}^{2}-\frac{\alpha_{2} x_{2} y}{1+\delta_{1} x_{1}+\delta_{2} x_{2}}=0 \\
& -\beta_{4} y+\frac{\left(\alpha_{1}^{\prime} x_{1} y+\alpha_{2} x_{2} y\right)}{1+\delta_{1} x_{1}+\delta_{2} x_{2}}=0
\end{aligned}
$$

Elimination of y from first and second equations of (3) gives the quadratic

$$
\alpha_{1} \beta_{1} x_{1}^{2}+\left(\alpha_{2} x_{2}-\alpha_{1} \beta_{2} x_{2}-\alpha_{1} \beta_{3} x_{2}^{2}\right) x_{1}-\alpha_{2} x_{2}^{2}=0
$$

The third equation of (3) gives:

$$
\begin{gathered}
\left(\alpha_{1}^{\prime}-\delta_{1} \beta_{4}\right) x_{1}+\left(\alpha_{2}-\delta_{2} \beta_{4}\right) x_{2}=\beta_{4} \\
P x_{1}+Q x_{2}=\beta_{4} ; P=\left(\alpha_{1}^{\prime}-\delta_{1} \beta_{4}\right), Q=\left(\alpha_{2}-\delta_{2} \beta_{4}\right)
\end{gathered}
$$

Now putting $x_{1}=\frac{\beta_{4}-Q x_{2}}{P}$ in equation (4), gives cubic equation in $x_{2}$

$$
f\left(x_{2}\right)=S_{0} x_{2}^{3}+S_{1} x_{2}^{2}+S_{2} x_{2}+S_{3}=0
$$

$S_{0}=\alpha_{1} \beta_{3} P Q ; S_{1}=\left[\alpha_{1} \beta_{1} Q^{2}-\alpha_{1} \beta_{3} \beta_{4} P-\alpha_{2} P^{2}+\left(\alpha_{1} \beta_{2}-\alpha_{2}\right) P Q\right] ; S_{2}=\left[P \beta_{4}\left(\alpha_{2}-\alpha_{1} \beta_{2}\right)-2 \alpha_{1} \beta_{1} \beta_{4} Q\right]$ $S_{3}=\alpha_{1} \beta_{1} \beta_{4}^{2} ; P=\alpha_{1}^{\prime}-\delta_{1} \beta_{4}, Q=\alpha_{2}-\delta_{2} \beta_{4}$

The y-coordinate from first and second equations of (3) is given as 


$$
\alpha_{1} x_{1} y=\left(x_{2}-x_{1}\right)\left(1+\delta_{1} x_{1}+\delta_{2} x_{2}\right) \text { or/And } \alpha_{2} x_{2} y=\left(\beta_{1} x_{1}-\beta_{2} x_{2}-\beta_{3} x_{2}^{2}\right)\left(1+\delta_{1} x_{1}+\delta_{2} x_{2}\right)
$$

From equation (5), no positive value of $x_{1}$ is possible for $P<0$ and $Q<0$. Considering, $P>0$ and $Q<0$, by Descartes rule of sign, the equation (6) will possess either one or three positive roots. Since $f(0)>0$ and $f\left(\beta_{4} / Q\right)<0$, there may exist one positive value of $x_{2}$. Further, $x_{1}$ and y may have one positive solution. Accordingly, one positive interior point may exist.

Considering, $P>0$ and $Q>0$, by Descartes rule, the cubic equation (6) admits only two positive roots. Now $f(0)>0$ and $f\left(\beta_{4} / Q\right)<0$. Consequently, $x_{2}$ has only one positive root in the interval $\left(0, \beta_{4} / Q\right)$. Further, only one positive value of $x_{1}$ is possible, provided $x_{2}<\beta_{4} / Q$ and y admits positive values, when equation (7) is satisfied. Combining these facts, the system admits only one equilibrium point in this case.

However, for $P<0, Q>0, x_{1}$ is positive for $x_{2}>\beta_{4} / Q$. Consequently, $f\left(x_{2}\right)$ admits at the most two positive roots in the interval $\left(\beta_{4} / Q, \infty\right)$. It is concluded that at the most two interior points are possible depending upon the condition (7).

\subsection{Local Stability Analysis}

The Jacobian matrix about any equilibrium point for the system (2) is computed as

$$
J=\left(a_{i j}\right)_{3 \times 3}=\left[\begin{array}{ccc}
-1-\frac{\alpha_{1} y\left(1+\delta_{2} x_{2}\right)}{\left(1+\delta_{1} x_{1}+\delta_{2} x_{2}\right)^{2}} & 1+\frac{\alpha_{1} \delta_{2} x_{1} y}{\left(1+\delta_{1} x_{1}+\delta_{2} x_{2}\right)^{2}} & -\frac{\alpha_{1} x_{1}}{\left(1+\delta_{1} x_{1}+\delta_{2} x_{2}\right)} \\
\beta_{1}+\frac{\alpha_{2} \delta_{1} x_{2} y}{\left(1+\delta_{1} x_{1}+\delta_{2} x_{2}\right)^{2}} & -\beta_{2}-2 \beta_{3} x_{2}-\frac{\alpha_{2} y\left(1+\delta_{1} x_{1}\right)}{\left(1+\delta_{1} x_{1}+\delta_{2} x_{2}\right)^{2}} & -\frac{\alpha_{2} x_{2}}{\left(1+\delta_{1} x_{1}+\delta_{2} x_{2}\right)} \\
\frac{\alpha_{1}^{\prime} y\left(1+\delta_{2} x_{2}\right)}{\left(1+\delta_{1} x_{1}+\delta_{2} x_{2}\right)^{2}} & \frac{\alpha_{2} y\left(1+\delta_{1} x_{1}\right)}{\left(1+\delta_{1} x_{1}+\delta_{2} x_{2}\right)^{2}} & -\beta_{4}+\frac{\left(\alpha_{1}^{\prime} x+\alpha_{2} x_{21}\right)}{\left(1+\delta_{1} x_{1}+\delta_{2} x_{2}\right)}
\end{array}\right]
$$

- The characteristic equation about $E_{0}=(0,0,0)$ is obtained as

$$
\left(\lambda+\beta_{4}\right)\left(\lambda^{2}+\left(1+\beta_{2}\right) \lambda+\left(\beta_{2}-\beta_{1}\right)\right)=0
$$

Accordingly, the point $E_{0}$ is locally asymptotically stable for $\beta_{1}<\beta_{2}$ and saddle for $\beta_{1}>\beta_{2}$. There is a possibility of trans-critical bifurcation at $\beta_{1}=\beta_{2}$. It can be established using Sotomayor's Theorem [8].

- The characteristic equation about $E_{1}=\left(\frac{\beta_{1}-\beta_{2}}{\beta_{3}}, \frac{\beta_{1}-\beta_{2}}{\beta_{3}}, 0\right)$ is given by:

$$
\left(\lambda^{2}+\left(2 \beta_{1}-\beta_{2}+1\right) \lambda+\left(\beta_{1}-\beta_{2}\right)\right)\left(\lambda+\beta_{4}-\frac{\left(\alpha_{1}^{\prime}+\alpha_{2}\right)\left(\beta_{1}-\beta_{2}\right)}{\beta_{3}+\left(\delta_{1}+\delta_{2}\right)\left(\beta_{1}-\beta_{2}\right)}\right)=0
$$

The two of the eigenvalues about $E_{1}$ are negative while eigenvalues in y-direction is 


$$
\lambda_{3}=\frac{\left(\alpha_{1}^{\prime}+\alpha_{2}\right)\left(\beta_{1}-\beta_{2}\right)}{\beta_{3}+\left(\delta_{1}+\delta_{2}\right)\left(\beta_{1}-\beta_{2}\right)}-\beta_{4}
$$

Hence, the boundary equilibrium $E_{1}$ is locally asymptotically stable if

$$
\beta_{4}>\frac{\left(\alpha_{1}^{\prime}+\alpha_{2}\right)\left(\beta_{1}-\beta_{2}\right)}{\beta_{3}+\left(\delta_{1}+\delta_{2}\right)\left(\beta_{1}-\beta_{2}\right)}
$$

It will become saddle, if above condition is violated. The trans-critical bifurcation occurs at

$$
\beta_{3}=\frac{\left(\beta_{1}-\beta_{2}\right)\left\{\left(\alpha_{1}^{\prime}+\alpha_{2}\right)-\beta_{4}\left(\delta_{1}+\delta_{2}\right)\right\}}{\beta_{4}} ;\left(\alpha_{1}^{\prime}+\alpha_{2}\right)-\beta_{4}\left(\delta_{1}+\delta_{2}\right) \neq 0
$$

Note 1: There exists no periodic solution in $x_{1}-x_{2}$ plane.

Note 2: The point $E_{1}$ collides with $E_{0}$ when $\beta_{1}=\beta_{2}$. The system (2) admits trans-critical bifurcation about $E_{1}$ with respect to parameters $\beta_{1}, \beta_{2}, \beta_{4}, \alpha_{1}^{\prime}, \alpha_{2}, \delta_{1}$ and $\delta_{2}$, whose values can be evaluated from the expression (8). The Sotomayor's Theorem is applied to establish the trans-critical bifurcation.

The coefficients of Jacobian matrix about $E^{*}$, when exists, are given by:

$$
\begin{aligned}
& a_{11}=-1-\frac{\alpha_{1} y^{*}\left(1+\delta_{2} x_{2}^{*}\right)}{\left(1+\delta_{1} x_{1}^{*}+\delta_{2} x_{2}^{*}\right)^{2}}(<0), a_{12}=1+\frac{\alpha_{1} \delta_{2} x_{1}^{*} y^{*}}{\left(1+\delta_{1} x_{1}^{*}+\delta_{2} x_{2}^{*}\right)^{2}}(>0), a_{13}=-\frac{\alpha_{1} x_{1}^{*}}{\left(1+\delta_{1} x_{1}^{*}+\delta_{2} x_{2}^{*}\right)}(<0), \\
& a_{21}=\beta_{1}+\frac{\alpha_{2} \delta_{1} x_{2}^{*} y^{*}}{\left(1+\delta_{1} x_{1}^{*}+\delta_{2} x_{2}^{*}\right)^{2}}(>0), a_{22}=-\beta_{2}-2 \beta_{3} x_{2}^{*}-\frac{\alpha_{2} y^{*}\left(1+\delta_{1} x_{1}^{*}\right)}{\left(1+\delta_{1} x_{1}^{*}+\delta_{2} x_{2}^{*}\right)^{2}}(<0), a_{33}=0 \\
& a_{23}=-\frac{\alpha_{2} x_{2}^{*}}{\left(1+\delta_{1} x_{1}^{*}+\delta_{2} x_{2}^{*}\right)}(<0), a_{31}=\frac{\alpha_{1}^{\prime} y^{*}\left(1+\delta_{2} x_{2}^{*}\right)}{\left(1+\delta_{1} x_{1}^{*}+\delta_{2} x_{2}^{*}\right)^{2}}(>0), a_{32}=\frac{\alpha_{2} y^{*}\left(1+\delta_{1} x_{1}^{*}\right)}{\left(1+\delta_{1} x_{1}^{*}+\delta_{2} x_{2}^{*}\right)^{2}}(>0)
\end{aligned}
$$

The characteristic equation about $E^{*}$ is $\left(\lambda^{3}+A_{0} \lambda^{2}+A_{1} \lambda+A_{2}\right)=0$

where

$$
\begin{aligned}
& A_{0}=-\left(a_{11}+a_{22}\right), A_{1}=a_{11} a_{22}-a_{12} a_{21}-a_{13} a_{31}-a_{23} a_{32} \\
& A_{2}=a_{11} a_{23} a_{32}-a_{12} a_{23} a_{31}-a_{13} a_{21} a_{32}+a_{22} a_{13} a_{31}
\end{aligned}
$$

It is clear that, $A_{0}>0$ and $A_{2}>0$. By Routh-Hurwitz criteria, interior point is locally asymptotically stable if $A_{0} A_{1}-A_{2}>0$.

Analytically the above condition could not be examined further. However, numerically it is verified for certain choice of parameters.

\subsection{Global Stability Analysis}


In this subsection, global stability of non-negative equilibrium points is investigated by choosing a suitable Lyapunov function.

Theorem 4.3.1: The trivial point $E_{0}=(0,0,0)$ is globally asymptotically stable for $\beta_{1}<\frac{\alpha_{1}^{\prime}}{\alpha_{1}}<\beta_{2}$.

Proof: Consider a Lyapunov function

$$
V_{1}=\sum_{i=1}^{2} K_{i} x_{i}+K_{3} y
$$

where, $K_{i}, i=1,2,3$ are the positive constants

After calculating the derivative $\dot{V}_{1}$ along each solution of system (2), we get

$$
\begin{aligned}
\dot{V}_{1}= & -\left(K_{1}-K_{2} \beta_{1}\right) x_{1}+\left(K_{1}-K_{2} \beta_{2}\right) x_{2}-K_{2} \beta_{3} x_{2}^{2}-K_{3} \beta_{4} y+\left(K_{3} \alpha_{1}^{\prime}-K_{1} \alpha_{1}\right) \frac{x_{1} y}{\left(1+\delta_{1} x_{1}+\delta_{2} x_{2}\right)} \\
& +\left(K_{3}-K_{2}\right) \frac{\alpha_{2} x_{2} y}{\left(1+\delta_{1} x_{1}+\delta_{2} x_{2}\right)}
\end{aligned}
$$

Let $K_{3} \alpha_{1}^{\prime}=K_{1} \alpha_{1}$ and $K_{3}=K_{2}$, then $\dot{V}_{1}=-\left(K_{1}-K_{2} \beta_{1}\right) x_{1}+\left(K_{1}-K_{2} \beta_{2}\right) x_{2}-K_{2} \beta_{3} x_{2}^{2}-K_{3} \beta_{4} y$

The derivative $\dot{V}_{1}<0$, if $\frac{K_{1}}{K_{2}}>\beta_{1}$ and $\frac{K_{1}}{K_{2}}<\beta_{2} \Rightarrow \beta_{1}<\frac{\alpha_{1}^{\prime}}{\alpha_{1}}<\beta_{2}$. Hence origin is globally asymptotically stable for $\beta_{1}<\frac{\alpha_{1}^{\prime}}{\alpha_{1}}<\beta_{2}$.

Theorem 4.3.2: The boundary point $E_{1}=\left(\frac{\beta_{1}-\beta_{2}}{\beta_{3}}, \frac{\beta_{1}-\beta_{2}}{\beta_{3}}, 0\right)$ is globally asymptotically stable in the positive quadrant of $x_{1} x_{2}$ plane.

Proof: Consider a Lyapunov function

$$
V_{2}=\sum_{i=1}^{2} K_{i}\left(x_{i}-\bar{x}_{i}-\ln \frac{x_{i}}{\bar{x}_{i}}\right) ; K_{1} \text { and } K_{2} \text { are the positive constants }
$$

The derivative $\dot{V}_{2}$ along each solution of system (2) gives

$$
\dot{V}_{2}=-\frac{K_{1} x_{2}\left(x_{1}-\bar{x}_{1}\right)^{2}}{x_{1} \bar{x}_{1}}+\left(x_{1}-\bar{x}_{1}\right)\left(x_{2}-\bar{x}_{2}\right)\left(\frac{K_{1}}{\bar{x}_{1}}+\frac{K_{2} \beta_{1}}{\bar{x}_{2}}\right)-\frac{K_{2} \beta_{1} x_{1}\left(x_{2}-\bar{x}_{2}\right)^{2}}{x_{2} \bar{x}_{2}}-K_{2} \beta_{3}\left(x_{2}-\bar{x}_{2}\right)^{2}
$$

Let $K_{1}=K_{2} \beta_{1}$

$$
\dot{V}_{2}<-\frac{K_{1}}{\bar{x}_{1}}\left[\sqrt{\frac{x_{2}}{x_{1}}}\left(x_{1}-\bar{x}_{1}\right)-\sqrt{\frac{x_{1}}{x_{2}}}\left(x_{2}-\bar{x}_{2}\right)\right]^{2}-\frac{K_{1} \beta_{3}}{\beta_{1}}\left(x_{2}-\bar{x}_{2}\right)^{2}
$$


Since, time derivative is negative definite, the boundary equilibrium point is globally asymptotically stable in the positive quadrant of $x_{1} x_{2}$-plane.

\section{Persistence}

The system is said to be persist, if all the populations survive for a long time.

Theorem 5.1: In the absence of nontrivial periodic solution in $x_{1}-x_{2}$ plane, the system (2) persists, if

$$
\lambda_{3}=\frac{\left(\alpha_{1}^{\prime}+\alpha_{2}\right)\left(\beta_{1}-\beta_{2}\right)}{\beta_{3}+\left(\delta_{1}+\delta_{2}\right)\left(\beta_{1}-\beta_{2}\right)}-\beta_{4}>0 \text { for } \beta_{1}>\beta_{2}
$$

For $\beta_{1}>\beta_{2}, E_{0}$ is unstable and the planar point exists. No periodic solution is possible under (9). Further, all trajectories near $x_{1}-x_{2}$ plane will be repelled and the solution will remain in the interior of $R_{+}^{3}$.

\section{Numerical Simulation}

Consider the data set:

$$
\alpha_{1}=0.2, \alpha_{2}=0.3, \alpha_{1}^{\prime}=0.4, \beta_{1}=0.5, \beta_{2}=0.22, \beta_{3}=0.35, \beta_{4}=0.15, \delta_{1}=0.5, \delta_{2}=0.25
$$

For this data set, $P>0, Q>0$, then the interior point $E^{*}$ becomes $(0.244909,0.268207,0.56578)$.At this $A_{0} A_{1}-A_{2}>0$. The fig. 1(a) shows the local stability of interior point.

The Hopf bifurcation is investigated with respect to parameter $\alpha_{1}^{\prime}$ and it occurs in the neighbourhood of $\alpha_{1}^{\prime} \approx 4.8085$. At $\alpha_{1}^{\prime} \approx 4.8084903$, the interior point becomes $E^{*} \approx(0.029808,0.033929,0.70747)$ and the corresponding eigenvalues are $-1.58567, \pm(0.166065)$ i. Also, $A_{0} A_{1}-A_{2} \approx 0$ and the first Lyapunov coefficient is computed as $-1.097495 \mathrm{e}-003$. The fig.1 (b) shows the existence of Hopf bifurcation.

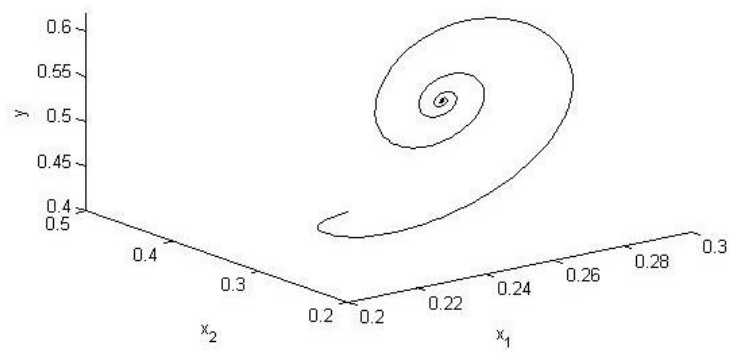

(a)

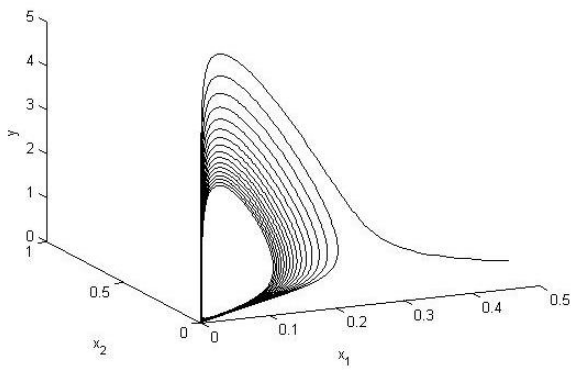

(b)

Fig. 1. Phase-portraits showing (a) local asymptotically stability behavior of $E^{*}$ and (b) existence of Hopf bifurcation at $\alpha_{1}^{\prime} \approx 4.8084903$.

Now, consider the case, $P<0, Q>0$, which is possible for the following choice of data:

$$
\alpha_{1}=0.2, \alpha_{2}=0.3, \alpha_{1}^{\prime}=0.06, \beta_{1}=0.5, \beta_{2}=0.35, \beta_{3}=0.22, \beta_{4}=0.15, \delta_{1}=0.5, \delta_{2}=0.25
$$


The polynomial $f\left(x_{2}\right)$ has three positive roots, which are $x_{21} \approx 0.486266, x_{22} \approx 0.60575$ and $x_{23} \approx$ 44.0898. Now, $x_{1}<0$ for $x_{21}$ and corresponding to $x_{22}$ and $x_{23}$, the value of $x_{1}$ is obtained as $x_{12} \approx$ 0.600719 and $x_{13} \approx 761.5714$ respectively. Also, $y<0$ for $x_{2}=x_{23}$. In this case, unique interior point $E^{*} \approx$ (0.60072, 0.60575, 0.06086) exists and found to be stable, since $A_{0} A_{1}-A_{2}>0$. The fig.2 (a) verifies its stability.

However, the Hopf bifurcation occurs at $\alpha_{1}^{\prime} \approx 5.7291806$, the interior point will be $E^{*} \approx(0.02527$, $0.027121,0.373455)$ and the corresponding eigenvalues are $-1.54346, \pm(0.120576)$ i. The first Lyapunov coefficient is computed as $-1.050622 \mathrm{e}-003$. The existence of Hopf bifurcation can be seen from fig.2 (b).

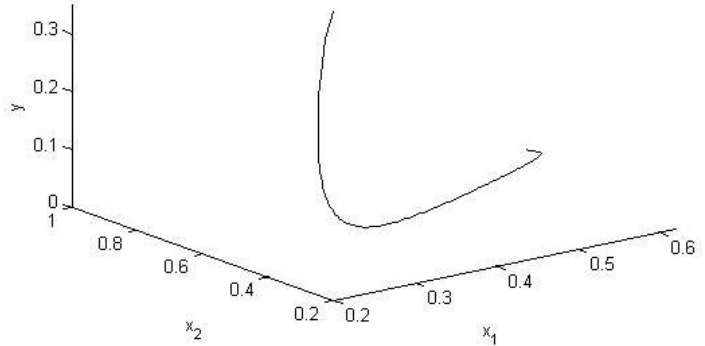

(a)

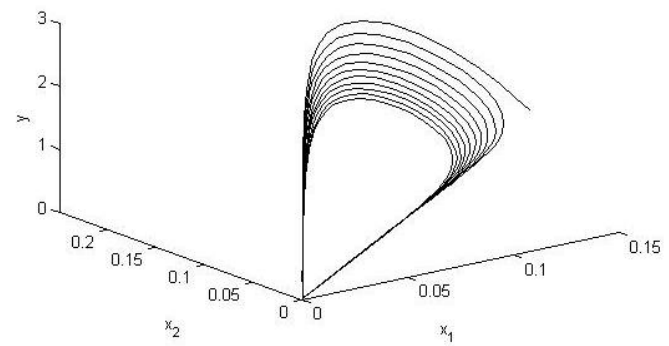

(b)

Fig. 2. Phase-portraits showing (a) stability behavior of $E^{*}$ and (b) existence of Hopf bifurcation at $\alpha_{1}^{\prime} \approx$ 5.7291806

\section{Conclusion}

In this paper, a stage-structured predator-prey system is proposed, where both immature and mature prey are taken by the predator. Two types of bifurcations are investigated; trans-critical bifurcation about trivial and planar points and Hopf bifurcation about interior point with respect to some parameter. It is observed that, if predator is not getting enough energy from immature prey, so that conversion factor $\alpha_{1}^{\prime} \approx 0$, then the chances of co-existence of species will be less. It is clear that food taken from juvenile prey may be crucial for the survival of predator.

\section{Acknowledgment}

The second author (K. Gupta) would like to thank University Grants Commission for providing Senior Research Fellowship through Grant No. 6405-11-044.

\section{References}

[1] Devi, S. (2013). Effects of prey refuge on a ratio-dependent predator-prey model with stage-structure of prey population. Applied Mathematical Modelling, 37(6), 4337-4349.

[2] Kaili, Y., \& Xinyu, S. (2003). Predator-prey system with stage structure and delay. Applied Mathematics-A Journal of Chinese Universities, 16(2), 143-150.

[3] $\mathrm{Xu}, \mathrm{S}$. (2014). The dynamics of a general prey-predator model with prey-stage structure and diffusive effects. Computer and Mathematics with Applications, 68(3), 405-423.

[4] Zhang, L., \& Zhang, C. (2010). Rich dynamic of a stage-structured prey-predator model with cannibalism and periodic attacking rate. Communication Nonlinear Science and Numerical Simulation, $15,4029-4040$. 
[5] Zhang, X., Chen, L., \& Neumann, A. U. (2000). The stage-structured predator-prey model and optimal harvesting policy. Mathematical Biosciences, 168, 201-210.

[6] Falconi, M., Huenchucona, M., \& Vidal, C. (2015). Stability and global dynamic of a stage-structured predator-prey model with group defense mechanism of the prey. Applied Mathematics and Computation, 270, 47-61.

[7] Yang, L., \& Zhong, S. (2014). Global stability of a stage-structured predator-prey model with stochastic perturbation. Discrete Dynamics in Nature and Society.

[8] Perko, L. (1991). Differential Equations and Dynamical Systems. Springer, New York.

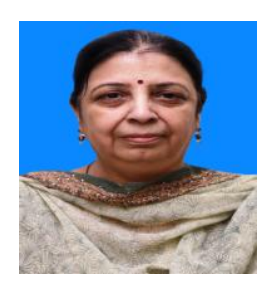

Sunita Gakkhar has completed her Ph.D. from Indian Institute of Technology Kanpur, Uttar-Pradesh, India. She is working as a professor at the Department of Mathematics, Indian Institute of Technology Roorkee, Uttarakhand, India. Her area of interest is bio mathematics.

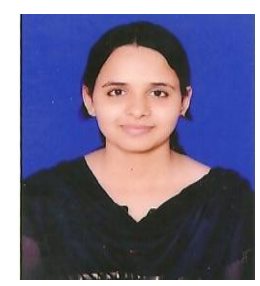

Komal Gupta is a research scholar in the Department of Mathematics, Indian Institute of Technology Roorkee, Uttarakhand, India. Her area of interest is dynamical systems. 